\title{
N-SUBSTITUTION AND $\alpha_{1}$-ADRENERGIC RECEPTOR AFFINITY OF LAUDANOSINE ANALOGUES
}

\author{
PATRICIO ITURRIAGA-VÁSQUEZ, BRUCE K. CASSELS, ,† \\ M. DOLORES IVORRA AND M. PILAR D'OCON:
}

\begin{abstract}
Millennium Institute for Advanced Research in Cell Biology and Biotechnology and Departamento de Química, Facultad de Ciencias, Universidad de
\end{abstract} Chile, Santiago, Chile, and Departament de Farmacologia, Facultat de Farmàcia, Universitat de Valencia, Valencia, Spain.

\begin{abstract}
Benzyltetrahydroisoquinoline (BTHIQ) molecules are able to adopt widely differing conformations that depend on the presence or absence of $N$-substituents. To assess the possible role of BTHIQ conformation on the affinity of these compounds for $a_{1}$-adrenergic receptors, of interest for the management of hypertension, the racemic $N$-unsubstituted BTHIQ norlaudanosine and a series of $N$-alkylated derivatives were assessed for binding to rat brain cortical sites labelled with the radioligand $\left[{ }^{3} \mathrm{H}\right]$ prazosin. The $\alpha_{1}$-adrenergic affinity in this series increased with the bulk of the substituent on the nitrogen atom, from the $N$-ethyl to the $N$-propyl analogue. Comparison of these results with published data for related BTHIQs and for the rigid mimics of the fully extended and semi-folded conformations of laudanosine, tetrahydropalmatine and glaucine, suggested that the $\alpha_{1}$-adrenergic receptor binding site is able to accommodate either conformation. The presence of a bulky substituent on the nitrogen atom seems to favor receptor binding independently of the favored conformation, and that the orientation in which BTHIQs are bound probably differs depending on the presence or absence of a hydroxyl group at a key position.
\end{abstract}

Keywords: $\alpha_{1}$-adrenergic receptor binding; norlaudanosine; laudanosine; $N$-alkylnorlaudanosines; benzyltetrahydroisoquinolines; tetrahydropalmatine; glaucine

\section{INTRODUCTION}

Laudanosine (2) is a minor benzyltetrahydroisoquinoline (BTHIQ) alkaloidal constituent of opium, ${ }^{1}$ and a major metabolite of the clinically important skeletal muscle relaxant atracurium, ${ }^{2}$ as well as of its pure stereoisomer cisatracurium, both of which are used in combination with general anesthetics. One of the many pharmacological actions of laudanosine that may be of clinical concern in anesthesia is the relaxation of smooth muscle, mainly via blockade of $a_{1}$-adrenergic receptors. ${ }^{3}$ On the other hand, subtype-selective $a_{1}$-adrenergic antagonism is of interest as an approach to the management of hypertension, and a greater understanding of the structural features leading to subtype selectivity is a prerequisite for the development of useful drugs.

We have recently shown that $a_{1}$-adrenergic antagonism also operates in the case of the related BTHIQ coclaurine and some of its derivatives, although sometimes competing with $\mathrm{Ca}^{2+}$ entry blockade which also leads to a loss of vascular tone and thus to a reduction in arterial blood pressure. ${ }^{4}$ In the latter series, substitution on the nitrogen atom with a methyl or ethyl group resulted in small changes in $a_{1}$-adrenergic receptor affinity without any general trend emerging, although in the coclaurine and norarmepavine families, bearing a para hydroxyl group on the pendent benzyl moiety, $N$-substitution led to decreased affinities in the order $\mathrm{H}>\mathrm{CH}_{3}>\mathrm{CH}_{2} \mathrm{CH}_{3}$.

BTHIQs are flexible molecules that are able to adopt three main conformations: a completely extended one, a folded one, and one that we call "semi-folded". ${ }^{5}$ The $N$-unsubstituted secondary amine BTHIQs show a clear preference for the extended conformation, and the $\mathrm{N}$-alkylated analogues reside predominantly in the semi-folded conformation, as shown by NMR and molecular modeling studies. The extended and semi-folded conformations of BTHIQs approximate the molecular shapes of the berbine or tetrahydroberberine skeleton, and of aporphine alkaloids, respectively (Fig. 1). As a considerable number of these sterically constrained berbines and aporphines have also been shown to exhibit $a_{1}$-adrenergic receptor blocking activity, ${ }^{6,7}$ we decided to compare the affinities of laudanosine (2), norlaudanosine (1) and its $N$-ethyl (3) and -propyl (4) derivatives with the published data for their extended and semifolded rigid analogues, tetrahydropalmatine (5) and glaucine (6), and with our own results for the flexible coclaurine and armepavine analogues. ${ }^{4}$

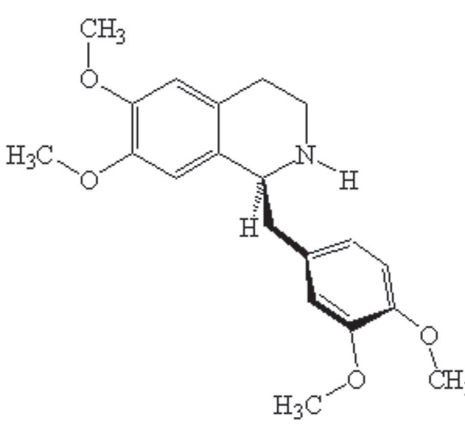

1<smiles>[R]N1CCc2cc(OC)c(OC)cc2[C@H]1Cc1ccc(OC)c(OC)c1</smiles>

2: $\mathrm{R}=\mathrm{CH}_{3}$

3: $\mathrm{R}=\mathrm{CH}_{2} \mathrm{CH}_{3}$

4: $\mathrm{R}=\mathrm{CH}_{2} \mathrm{CH}_{2} \mathrm{CH}_{3}$<smiles>COc1cc2c(cc1OC)CN1CCc3cc(OC)c(OC)cc3[C@H]1C2</smiles>

5<smiles>COc1cc2c(cc1OC)-c1c(OC)c(OC)cc3c1[C@H](C2)N(C)CC3</smiles>

6

Figure 1: Preferred conformations of norlaudanosine (1), laudanosine (2), $\mathrm{N}$-ethyl- and -propyl-norlaudanosine (3 and $\mathbf{4}$, respectively), showing their steric relationships to tetrahydropalmatine (5) and glaucine (6). 


\section{EXPERIMENTAL}

\section{General}

The following commercial drugs were used: prazosin and phentolamine were from Sigma (St. Louis, MO); $\left[{ }^{3} \mathrm{H}\right]$ prazosin $\left(20.3 \mathrm{Ci} \mathrm{mmol}^{-1}\right)$ was from Amersham International (Buckinghamshire, UK). Other reagents and solvents were of analytical grade. Norlaudanosine (1) and its derivatives (2-4) assayed in this work were prepared in our laboratory, and their physical, spectroscopic, and analytical data and conformational studies have been previously reported by us. ${ }^{5}$

\section{Binding studies}

Female Wistar rats, 180-200 g, were decapitated and the brain rapidly removed. The cerebral cortex was dissected and homogenized in 10 volumes (w/v) ice-cold buffer (Tris $\mathrm{HCl} 5 \mathrm{nM}$, sucrose $250 \mathrm{mM}$ and EDTA $1 \mathrm{mM}$; $\mathrm{pH}$ 7.5 at $\left.25^{\circ} \mathrm{C}\right)$ using an Ultra-Turrax $(3 \times 15 \mathrm{~s})$. The homogenate was centrifuged for $10 \mathrm{~min}$ at $1000 \times \mathrm{g}$, the pellet was discarded and the supernatant was centrifuged at $26000 \times \mathrm{g}$ for $15 \mathrm{~min}$ at $4{ }^{\circ} \mathrm{C}$. The final pellet was resuspended in assay buffer (Tris $\mathrm{HCl} 50 \mathrm{mM}, \mathrm{pH} 7.5$ at $25^{\circ} \mathrm{C}$ ) and stored at $-70^{\circ} \mathrm{C}$ for later use. All membrane preparation procedures were conducted at $4{ }^{\circ} \mathrm{C}$. Binding of $\left[{ }^{3} \mathrm{H}\right]$ prazosin to rat cerebral cortical membranes was saturable, reversible and showed high affinity, with a dissociation constant $K_{\mathrm{d}}=0.14 \mathrm{nM}$, and occurred at a single class of binding sites. ${ }^{8}$ The incubation volume was $1 \mathrm{ml}$ (approx. $250 \mu \mathrm{g}$ protein/tube). The assay tubes were incubated with $\left[{ }^{3} \mathrm{H}\right]$ prazosin $(0.1-$ $0.2 \mathrm{nM}$ ) in the absence or in the presence of drug at various concentrations. Incubations were carried out at $25{ }^{\circ} \mathrm{C}$ for $45 \mathrm{~min}$ and the binding reactions were then terminated by rapid vacuum filtration using a Brandel cell harvester (M24R) with fibre-glass filters (Schleicher and Schuell, $\mathrm{N}^{\circ} 30$ ) presoaked in $0.3 \%$ polyethyleneimine for $5 \mathrm{~min}$. The filters were then washed with ice-cold $50 \mathrm{mM}$ Tris-HCl buffer, $\mathrm{pH} 7.5(4 \times 4 \mathrm{ml})$ and the radioactivity bound to the filters was determined by liquid scintillation counting. Non-specific binding was determined in the presence of $10^{-6} \mathrm{M}$ phentolamine. Proteins were assayed according to the method of Bradford with $\gamma$-globulin as standard. ${ }^{9}$ All results were obtained in duplicate. Inhibition curves were analyzed by the weighted least-squares iterative Prism curve-fitting program (Graph Pad Software Inc., 2003), and inhibition constants $\left(K_{\mathrm{i}}\right)$ were calculated by use of the Cheng and Prussoff formula. ${ }^{10}$ Results are presented as mean \pm standard error of the mean (s.e.m.) of at least three experiments from at least two diferent batches of cerebral cortex. Where appropiate, one-way Anova test (available in Graph Pad Software Inc., 2003) for paired data was used, and values of $P<0.05$ were regarded as significant.

\section{RESULTS AND DISCUSSION}

The affinities of racemic norlaudanosine (1) and its $N$-methyl (laudanosine, 2), $N$-ethyl (3) and $N$-propyl (4) derivatives for $a_{1}$-adrenergic receptors was assessed in binding studies in rat brain cortical homogenates using the subtype-nonselective radioligand $\left[{ }^{3} \mathrm{H}\right]$ prazosin. The specific binding of this $\mathrm{a}_{-}$ adrenergic radioligand at a concentration of $0.2 \mathrm{nM}$ represented approximately $90 \%$ of the total binding. All the compounds inhibited $\left[{ }^{3} \mathrm{H}\right]$ prazosin binding with the inhibition constants $\left(K_{\mathrm{i}}\right)$ summarized in Table 1.

Table 1. Inhibition of $\left[{ }^{3} \mathrm{H}\right]$ prazosin binding to rat brain cortical sites by norlaudanosine (1), laudanosine (2), $\mathrm{N}$-ethyl- (3) and $\mathrm{N}$-propylnorlaudanosine $(4)^{\mathrm{a}}$

\begin{tabular}{|c|c|c|}
\hline Compound & $K_{\mathrm{i}}(\mu \mathrm{M})$ & Slope \\
\hline $\mathbf{1}$ & $22.65 \pm 0.67$ & 1.281 \\
\hline $\mathbf{2}$ & $17.76 \pm 0.28$ & 1.121 \\
\hline $\mathbf{3}$ & $11.68 \pm 0.22$ & 0.968 \\
\hline $\mathbf{4}$ & $5.05 \pm 0.09$ & 1.139 \\
\hline
\end{tabular}

a All binding experiments were carried out 3-5 times in duplicate. Differences are significant at the $P<0.001$ level (one-way Anova).
These inhibition constants are high (signifying low potency) when compared with values recorded for $a$-adrenergic blockers such as prazosin $(0.11 \mathrm{nM}),{ }^{11}$ but are quite comparable to those of related natural products (see below). To search for analogues with considerably higher affinities by introducing appropriate structural modifications on the easily accessible BTHIQ scaffold thus remains an attractive goal.

Norlaudanosine (1) and all its $N$-alkylated derivatives (2-4) bound to $a_{1}$-adrenergic receptors in a competitive manner and at a single site. Concentration-response curves are shown in Fig. 2. The rank order of affinities for norlaudanosine and its $N$-alkyl derivatives tested was $N$-propyl $>N$-ethyl $>N$-methyl $=$ norlaudanosine. Although the differences are not great, they are statistically significant. It is particularly noteworthy that the affinity of $\mathrm{N}$ propylnorlaudanosine is more than double that of its $N$-ethyl analogue, and almost four times that of laudanosine or norlaudanosine.

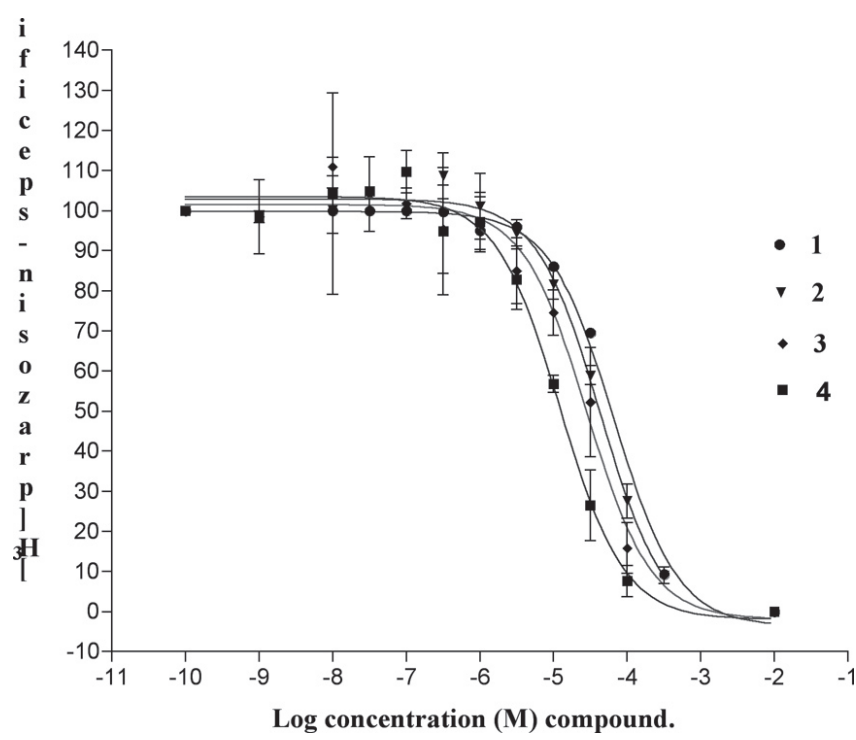

Figure 2. Inhibition of the specific binding of $\left[{ }^{3} \mathrm{H}\right]$ prazosin (molar concentration-response curves) to rat cerebral cortical membranes by racemic norlaudanosine (1), laudanosine (2) $N$-ethyl- (3) and $N$-propylnorlaudanosine (4). Each point is the mean of the results from three or five experiments performed in duplicate. All differences are significant at the $P<0.001$ level (Anova).

Based on earlier reports, we have established unambiguously that substitution of the secondary nitrogen of BTHIQs produces a shift in the conformational preference of the molecule, leading to a predominantly semifolded conformation in the $\mathrm{N}$-alkylated derivatives while secondary amines like norlaudanosine (1) prefer an extended conformation. A third, folded conformation, although not particularly unstable, is relatively inaccessible from the others due to the high potential energy barriers involved. ${ }^{5}$ Our experimental results, supported by theoretical calculations, showed that increasing the size of the BTHIQ $N$-substituent beyond methyl does not have any appreciable effect on the conformational equilibrium, and the only difference between the bulkier $\mathrm{N}$-alkyl and the $\mathrm{N}$-methyl derivatives should be kinetic, residing in the higher (i.e. $2.8 v s .1 \mathrm{kcal} / \mathrm{mole}$ ) energy barrier separating the accessible conformations in the former compounds. Therefore, the fact that the $a_{1}$-adrenergic receptor affinity of $N$-propylnorlaudanosine (4) is significantly higher than that of $N$ ethylnorlaudanosine (3) cannot be explained simply by the conformational preferences of these compounds, which are quite similar.

As the extended conformation of BTHIQs resembles the rigid shape of berbine alkaloids, while the semi-folded conformation approximates that of aporphines, a comparison of the affinities of the compounds studied here with those of closely related, tetramethoxylated berbines and aporphines seems warranted. The reported $K_{\mathrm{i}}$ value for the berbine tetrahydropalmatine (5) at

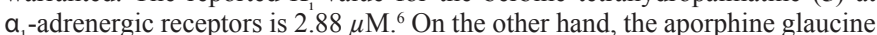
(6) also binds to these receptors with slightly greater affinity, and the inhibition 
curves displayed shallow slopes which could be subdivided into high and low affinity components attributed to $\mathrm{a}_{1 \mathrm{~A}^{-}}$and $\mathrm{a}_{1 \mathrm{~B}}$-receptor binding $\left(K_{\mathrm{i}}=0.07\right.$ and $1.20 \mu \mathrm{M}$, respectively). ${ }^{12}$ The rather small differences in the affinities of tetrahydropalmatine and glaucine, on one hand, and of the $\mathrm{N}$-unsubstituted and $N$-substituted BTHIQs, on the other, suggest that both the extended and the semifolded conformations can be accommodated by the ligand site of the $a_{1}$ adrenergic receptor.

It should be pointed out that the published affinities for tetrahydropalmatine and glaucine correspond to the natural $(S)$ isomers, while the compounds studied by us were racemic. Thus, if the $(S)$ stereochemistry were preferred (which is not known at this time), the $K_{\mathrm{i}}$ values recorded here for the laudanosine congeners might be half as high. In the case of $(S)$ - $N$-propylnorlaudanosine, its $a_{1}$-adrenergic receptor affinity would be slightly better than that reported for $(S)$-tetrahydropalmatine. Thus, although the $a_{1}$-adrenergic affinity of $( \pm)$ norlaudanosine and its $\mathrm{N}$-alkylated derivatives increases for the mostly semifolded $N$-ethyl- and -propyl compounds, the $K_{\mathrm{i}}$ values, particularly with regard to $(S)$-glaucine, do not clearly support the idea that these compounds mimic an aporphine-like conformation when bound to the receptor. It should be realized, however, that the biphenyl portion of the aporphine skeleton is practically flat (with the two coaxial benzene ring planes forming angles of less than $\left.30^{\circ}\right),{ }^{13}$ while the median plane of the benzyl group of BTHIQs in their semi-folded conformation is nearly perpendicular to the plane of the tetrahydroisoquinoline benzene ring. ${ }^{5}$ This difference may well be sufficient to explain the weaker affinity of the BTHIQs. As the proportion of molecules in the semi-folded conformation, barring specific interactions with the receptor, is not expected to differ to any important degree for the $N$-methyl, -ethyl or -propyl derivatives, the fact that the affinities of norlaudanosine (1) and laudanosine (2) are very similar and significantly lower than those of the compounds with larger substituents on the nitrogen atom demands another explanation. It seems reasonable to suggest that an ethyl or propyl group attached to the nitrogen atom may occupy a hydrophobic pocket in the binding site, rather like the $N$-propyl pocket described for $\mathrm{D}_{2}$-dopaminergic receptors, which have considerable structural homology with adrenergic receptors. A similar trend was seen for 12-O-methylcoclaurine and its $N$-methyl derivative, which exhibit $K_{\mathrm{i}}$ values of 12.02 and $5.12 \mu \mathrm{M}$, respectively, although at the time no conclusion could be drawn from these results. ${ }^{4}$ In the coclaurine (7,12-dihydroxy-6methoxyBTHIQ) series, however, $a_{1}$-adrenergic affinities decrease about twofold with each increasing carbon atom on going from the secondary amines to their $N$-methyl and ethyl analogues: the $K_{\mathrm{i}}$ values are 1.07, 2.34 and 5.13 $\mu \mathrm{M}$, respectively. In the norarmepavine (6,7-dimethoxy-12-hydroxyBTHIQ) series, the corresponding values are 5.89, 7.24 and $8.51 \mu \mathrm{M} .{ }^{4}$ The coclaurinenorarmepavine affinities run in the opposite direction to that observed in the present report with laudanosine and its analogues, and that reported by us in our 2003 paper for $12-O$-methylcoclaurine and its $\mathrm{N}$-methyl derivative. This suggests that BTHIQs bearing a hydroxyl group at the para position of the benzyl ring, such as coclaurine and armepavine on one hand, and analogues lacking this hydrogen-bond donor on the other, may bind to the receptor in different orientations. In both cases the BTHIQs presumably compete with norepinephrine for its binding site, but considering the likelihood that the receptor is able to accept ligands in extended or semifolded conformations, and in different orientations, extreme caution should be exercised before proposing any hypothetical pharmacophore.

\section{ACKNOWLEDGMENT}

This work was supported by ICM grant $\mathrm{N}^{\circ} \mathrm{P} 99-031-\mathrm{F}$ and by a research grant from the Generalitat Valenciana (GV01-292).

\section{REFERENCES}

1. V. Deulofeu, J. Comin and J. Vernengo, The benzylisoquinoline alkaloids. In: Manske, R. H. F. (Ed.), The Alkaloids. Academic Press: New York, NY, Vol. 10, pp 401-461, 1968.

2. V. Nigrovic and J. L. Fox, Atracurium decay and the formation of laudanosine in humans, Anesthesiology 74, 446-454 (1991).

3. S. Chuliá, M. D. Ivorra, C. Lugnier, E. Vila, M. A. Noguera and P. D'Ocon, Mechanism of the cardiovascular activity of laudanosine: comparison with papaverine and other benzylisoquinolines. Br. J. Pharmacol. 113, 13771385 (1994).

4. P. Iturriaga-Vásquez, R. Miquel, M. D. Ivorra, M. P. D’Ocon and B. K. Cassels, Simplified tetrandrine congeners as potential antihypertensives with a dual mechanism of action. J. Nat. Prod. 66, 954-957 (2003).

5. P. Iturriaga-Vásquez, G. Zapata-Torres, M. C. Rezende and B. K. Cassels, 1-Benzyl-1,2,3,4-tetrahydroisoquinolines. ${ }^{1} \mathrm{H}$ NMR conformational studies and rotational barriers. J. Chil. Chem. Soc. 49, 17- 23 (2004).

6. Z. Z. Lu, X. Wei, G. Z. Jin, and Q. D. Han, Antagonistic effect of tetrahydroproberberine homologues on alpha 1-adrenoceptor. Yao Xue Хиe Bao 31, 652-656 (1996), and work cited therein.

7. M. Valiente, P. D’Ocon, M. A. Noguera, B. K. Cassels, C. Lugnier, and M. D. Ivorra, Vascular activity of (-)-anonaine, (-)-roemerine and (-)-pukateine, three natural $6 \mathrm{a}(R)-1,2$ - methylenedioxyaporphines with different affinities for $\alpha_{1}$-adrenoceptor subtypes. Planta Med. 70, 603-609 (2004), and work cited therein.

8. S. Chuliá, J. Moreau, E. Naline, M. A. Noguera, M. D. Ivorra, M. P. D'O con and C. Advenier, The effect of S-(+)-boldine on the alpha 1 adrenoceptor of the guinea-pig aorta. Br. J. Pharmacol. 119, 1305-1312 (1996).

9. M. M. Bradford, A rapid and sensitive method for the quantitation of microgram quantities of protein utilizing the principle of protein-dye binding. Anal. Biochem. 72, 248-254 (1976).

10. Y. C. Cheng and W. H. Prusoff, Relationship between the inhibition constant $\left(\mathrm{K}_{\mathrm{i}}\right)$ and the concentration of inhibitor which causes 50 per cent inhibition $\left(\mathrm{I}_{50}\right)$ of an enzymatic reaction. Biochem. Pharmacol. 22, 30993108 (1973).

11. J. Sallés and A. Badía, Selective enrichment with $a_{1 \wedge}$ and $a_{18}$ adrenoceptor subtypes in rat brain cortical membranes. Eur. J. Pharmacol. 266, 301-308 (1994).

12. Y. Madrero, M. Elorriaga, S. Martínez, M. A. Noguera, B. K. Cassels, P. D'Ocon and M. D. Ivorra, A possible structural determinant of selectivity of boldine and derivatives for the $a_{1}$-adrenoceptor subtype. $B r . \quad J$. Pharmacol. 119, 1563-1568 (1996).

13. M. Asencio, B. K. Cassels, V. Manríquez and D. Boys, Structure of $(S) 1,10-$ dimethoxy-2,9-dihydroxyaporphinium chloride (boldine hydrochloride). Acta Cryst. C52, 1581-1583 (1996). 